\title{
Estudio multicéntrico sobre la calidad asistencial percibida por los pacientes en programa de hemodiálisis de Tarragona
}

\author{
Esperança Anguera Guinovart, Francisca Martínez Bateman
}

Enfermeras del Hospital Universitario Joan XXIII de Tarragona

\section{Resumen}

Las nuevas tecnologías han optimizado el tratamiento renal sustitutivo, sin embargo, nuestro interés se centra en conocer la opinión del paciente sobre los cuidados que recibe en las Unidades de Hemodiálisis (HD), con el fin de mejorar la asistencia sanitaria. El objetivo del estudio es determinar la calidad asistencial percibida por el usuario en las Unidades de Hemodiálisis de la provincia de Tarragona.

Se pretende encuestar a los pacientes de los centros de HD de la provincia de Tarragona con Insuficiencia Renal Crónica que deseen participar en el estudio. Se utiliza la encuesta SERVQHOS modificada y la escala Likert para determinar el Índice de Satisfacción del usuario. Hemos ponderado los resultados mediante dos métodos distintos: La correlación y la Ponderación del paciente para determinar cuál es más fiable. Se realiza el tratamiento informático de los datos con el programa informático SPSS-15 para Windows.

Según los resultados obtenidos, la Satisfacción Global del Paciente es de 4.3 sobre 5 (muy satisfecho) Alpha de Cronbach de 0.90. Los ítems del cuestionario que mayor importancia tienen para el

Correspondencia:

Esperança Anguera Guinovart

Vidal i Barraquer, $97^{\circ}-\mathrm{A}$

43005 TARRAGONA

e-mail: espean@tinet.org paciente son $(p<0.05)$. La disposición del personal para ayudar, el interés del personal para solucionar problemas, la confianza y seguridad que el personal transmite, la amabilidad del personal, la preparación del personal, el trato personalizado que se da a los pacientes y el interés del personal de enfermería. Los Índices de Satisfacción Ponderada de los tres centros son: Centro A: 8, Centro B: 8.6, Centro C: 8. Los usuarios más satisfechos se encuentran en el centro B. Verificamos que la ponderación de paciente es un procedimiento más fiable que la ponderación por correlación. Se han detectado oportunidades de mejora de la calidad asistencial.

Mediante el estudio multivariante hemos comprobado que las Características de los centros influyen en 8 de los ítems de Satisfacción $(2,3,5,9,13$, $14,19,21)(p<0.05)$. También hemos comprobado que 16 Características de los pacientes influyen en 18 de los ítems de Satisfacción de la encuesta $(p<0.05)$ (todos excepto $1,10,12)$. Por último, sería interesante hacer un estudio de tipo cualitativo ya que intuimos que la satisfacción del usuario puede depender, en gran medida, de la relación de ayuda que se establece con la enfermera.

\section{PALABRAS CLAVE:}

- HEMODIÁLISIS

- CALIDAD ASISTENCIAL PERCIBIDA

- CUESTIONARIO SERVQHOS

- SATISFACCIÓN PONDERADA POR EL PACIENTE 


\section{Multicentric study of the care quality perceived by patients on haemodialysis programme in Tarragona}

\begin{abstract}
:
New technologies have optimized renal replacement therapy. However, our interest is focused on finding out the patient's opinion of the care received at Haemodialysis (HD) Units, in order to improve the healthcare. The aim of the study is to determine the care quality perceived by the user in the Haemodialysis Units in the province of Tarragona.
\end{abstract}

It is sought to survey patients of the HD centres in the province of Tarragona with Chronic Renal Insufficiency who wish to participate in the study. The modified SERVQHOS survey and the Likert scale are used to determine the user Satisfaction Index. We have weighted the results using two different methods: correlation and Weighting of the patient to determine which is more reliable. The data were processed using the SPSS-15 programme for Windows.

According to the results obtained, Overall Patient Satisfaction is 4.3 out of 5 (very satisfied) Cronbach's alpha 0.90 . The items on the survey that are most important to patients are $(p<0.05)$ Staff's willingness to help, Staff's interest in solving problems, The confidence and assurance conveyed by staff, The friendliness of staff, How well-trained staff are, The personalized way patients are treated, and Nursing staff's interest. The Weighted Satisfaction Indices for the three centres are: Centre A: 8 , Centre B: 8.6, Centre $C: 8$. The most satisfied users are found in centre $B$. We verified that patient weighting is a more reliable procedure than correlation weighting. Opportunities for improvement in care quality have been detected. Using the multivariate study we have confirmed that the Characteristics of the centre affect 8 of the Satisfaction items $(2,3,5,9,13$, $14,19,21)(p<0.05)$. We have also found that 16 Patient Characteristics affect 18 of the Satisfaction items on the survey $(p<0.05)$ (all except $1,10,12)$. Finally, it would be interesting to carry out a qualitative study as we sense that user satisfaction may depend, to a significant extent, on the relationship of aid established with the nurse.

\section{KEY WORDS:}

- HAEMODIALYSIS

- PERCEIVED CARE QUALITY

- SERVQHOS SURVEY

- WEIGHTED PATIENT SATISFACTION

\section{Introducción}

La mayoría de nuestros usuarios son pacientes crónicos diagnosticados de una patología incurable, de larga duración y que requiere tratamiento periódico y continuado para asegurar su supervivencia.

En este contexto, los cuidados de enfermería han ido cambiando a medida que variaba el concepto de salud: medicina tecnológica, afectiva, integral, paternalista...

Como dijo Albert Jovell en su conferencia Declaración de Barcelona y Foro Español de Pacientes: "Desde hace un tiempo comenzamos a ver a los pacientes como Clientes, hecho que implica otros derechos y otras exigencias. Si asumen el rol de consumidor y cliente, claramente tendrán que tener la capacidad de elección y decisión".

He aquí la importancia de acercarnos al paciente y conocer sus inquietudes y opiniones e intentar mejorar nuestras actuaciones en función de sus expectativas.

Nos hemos propuesto evaluar la calidad asistencial percibida por el usuario en las Unidades de Hemodiálisis, ya sea por motivos éticos, de seguridad, de responsabilidad, también por motivos económicos, personales o legales, o simplemente por querer hacer bien nuestro trabajo.

Para ello necesitamos conceptualizar la palabra Calidad, que según Montgomery (1985) se define como el grado en que los productos cumplen con las expectativas de los clientes. Es importante tener claro que no es lo mismo Calidad prestada por unos profesionales esforzados en conseguirlo, que Calidad recibida por unos usuarios con las expectativas puestas en obtener la máxima calidad de los de cuidados que recibe ${ }^{1}$. La calidad varía según la contemplemos: desde el punto de vista del usuario, del profesional asistencial, o del gestor que administra los recursos ${ }^{2}$. Nosotros nos centraremos en La Calidad Percibida por el Usuario que viene determinada por 
la Satisfacción del paciente. Teniendo en cuenta que la tendencia actual es considerar la Satisfacción del paciente, como el resultado de comparar la evaluación que hace el paciente sobre la atención sanitaria recibida, con sus expectativas previas de una atención mínimamente aceptable 3 .

Determinar el Índice de Satisfacción Global (ISG) del usuario con el servicio, actualizado de forma periódica nos permitirá obtener una evaluación continua de la satisfacción de nuestros pacientes, comparable periodo tras periodo.

\section{Objetivos:}

\section{Objetivo principal}

- determinar la calidad percibida por el paciente con Insuficiencia Renal Crónica (IRC) incluido en programa de Hemodiálisis en la provincia de Tarragona.

\section{Objetivos secundarios}

- Determinar el perfil del paciente incluido en programa de Hemodiálisis.

- Determinar el perfil de centro de Hemodiálisis en la provincia de Tarragona.

- Hallar el Índice de Satisfacción de cada ítem de la encuesta.

- Determinar qué aspectos del cuestionario son más importantes para el paciente.

- Detectar áreas de mejora.

- Comprobar si la ponderación de los ítems del cuestionario por el propio paciente, refleja mejor su satisfacción, que la ponderación por coeficientes de correlación.

- Relacionar el grado de satisfacción con distintas variables: sociodemográficas, características del propio paciente, características propias de la diálisis o características del centro.

\section{Material y métodos:}

Estudio de investigación de resultados de enfermería, multicéntrico y transversal. Se trata de un estudio descriptivo y correlacional, realizado a 148 pacientes sometidos a programa de HD. Realizamos el estudio en 3 de los 5 centros de la provincia de Tarragona. Debido a un problema de salud acontecido en uno de los centros, no obtuvimos el permiso para incluir ni a dicho centro ni a otro de la misma empresa. Los centros participantes son: Unidad de Diálisis del Hospital Joan XXIII de Tarragona, Unidad de Diálisis del Hospital de la Santa Creu de Jesús de Tortosa y Unidad de Diálisis del Pius Hospital de Valls. Los datos fueron recogidos del 11 de mayo al 30 de agosto de 2010.

Incluimos a todos aquellos pacientes con IRC en programa de Hemodiálisis residentes en la provincia de Tarragona de los centros mencionados, que llevaban más de un mes en programa de Hemodiálisis (HD) y que reunían las condiciones físicas, mentales e idiomáticas para rellenar el cuestionario. Todos los pacientes participantes firmaron el consentimiento informado.

Como instrumento de medida hemos usado la encuesta SERVQHOS. Para la recogida de datos se diseñó un cuestionario:

La primera parte incluye Características de los pacientes, divididas en datos socio-demográficos: edad, sexo, estado civil, distancia entre residencia y centro de HD, nivel de estudios, situación laboral y datos clínicos: tiempo en programa de Hemodiálisis, acceso vascular, turno de HD, trasplantes. Estos datos permiten determinar el perfil del paciente.

Hemos incluido cuatro preguntas directas ya que éstas se han confirmado en numerosos estudios como criterios predictores de calidad percibida ${ }^{3,4}$. La primera sobre el nivel de satisfacción global con el servicio de Hemodiálisis, la segunda hace referencia a si el paciente recomendaría el servicio a otras personas, la tercera y cuarta pregunta responden a si el paciente conoce el nombre del médico y si conoce el nombre de la enfermera que le atiende.

En la segunda parte hemos utilizado el instrumento que nos permite evaluar la calidad percibida por el paciente, el cuestionario SERVQHOS, referente para estudios de satisfacción de los usuarios de Servicios Sanitarios, que combina expectativas y percepciones. Se trata de un cuestionario ya validado, que ha demostrado una alta consistencia interna, una buena capacidad predictiva, y especialmente una buena estructura factorial, ha demostrado discriminar entre pacientes satisfechos e insatisfechos y además nos permite identificar oportunidades de mejora ${ }^{5}$. Se trata de puntuar 21 ítems, agrupados en una dimensión objetiva (enumerados del 0 al 10 ) referentes a aspectos tangibles y otra dimensión subjetiva (enumerados del 11 al 21), referentes a cortesía, empatía, capacidad de respuesta y competencia profesional etc. 


\begin{tabular}{|l|l|}
\hline \multicolumn{1}{|c|}{ CALIDAD OBJETIVA } & \multicolumn{1}{c|}{ CALIDAD SUBJETIVA } \\
\hline 1.Tecnología de los equipos médicos & 11.Rapidez de respuesta \\
\hline 2.Apariencia del personal & 12.La disposición para la ayuda \\
\hline 3.Señalización llegar a Hemodiálisis & 13.Interés por solucionar problemas \\
\hline 4.Servicio de transporte & 14.Confianza y seguridad del personal \\
\hline 5.Puntualidad de las sesiones de HD & 15.Amabilidad del personal \\
\hline 6.Tiempo de espera atención medica & 16.Interés por cumplir promesas \\
\hline 7.Información que da el medico & 17.Preparación del personal \\
\hline 8.Información a familiares & 18.Trato personalizado \\
\hline 9.Información que da enfermería & 19.Capacidad comprender necesidades \\
\hline 10.Comodidad de las instalaciones & 20.Interés del personal de enfermería \\
\hline & 21.El respeto a la intimidad \\
\hline
\end{tabular}

Tabla 1. Tabla $n^{\circ}$ I. A partir de SERVQHOS. Cuestionario para evaluar la calidad percibida de la atención hospitalaria"111. Refleja las dos dimensiones de nuestro cuestionario adaptado.

Cada ítem se puntúa con escala Likert, desde 1 ("mucho peor de lo que esperaba") hasta 5 ("mucho mejor de lo que esperaba"). Hemos modificado el cuestionario SERVQHOS, para adaptarlo a nuestro estudio y a las salas de Hemodiálisis, añadiendo 2 ítems relacionados con: Ia Información que da enfermería y El respeto a la intimidad del paciente. Del mismo modo hemos sustituido una pregunta sobre Como llegar al hospital por otra referente al Servicio de transporte.

Al realizar la revisión bibliográfica, durante la planificación del estudio, nos pareció imprescindible ponderar los resultados en función de la importancia que cada ítem tiene para el paciente, ya que no es lo mismo puntuar alto un ítem poco importante para el paciente que puntuar alto un ítem muy importante para el paciente ${ }^{7,8}$. Referente a esto, queremos apuntar que los aspectos que el paciente puntúe como poco importantes no influirán tanto en el grado de satisfacción como aquellos ítems que el paciente considere muy importantes. Para calcular el Índice Ponderado de Satisfacción del usuario hemos usado dos métodos distintos y los hemos comparado entre sí: la ponderación calculada por coeficientes de correlación y la ponderación calculada en función de la importancia directamente atribuida por el usuario a cada aspecto del servicio ${ }^{7}$, para ello hemos incluido en el cuestionario una tabla de ponderación que permite puntuar, del 1 al 10, cada ítem del cuestionario SERVQHOS según la importancia que el paciente da a cada aspecto, los pacientes rellenaron esta tabla antes de cumplimentar el cuestionario.
Para completar la información hemos añadido tres preguntas abiertas, donde el usuario debe identificar los tres aspectos más negativos de la atención que recibe y los tres aspectos más positivos. El paciente puede añadir sugerencias libres.

Análisis de los datos Se introdujeron los datos en el programa Excel para realizar el estudio descriptivo. Para llevar a cabo el estudio multivariante se utilizó el programa estadístico SPSS 15 para Windows. Se codificaron e introdujeron las respuestas de cada paciente diferenciándolas por centros. Se realizó un estudio descriptivo de la muestra utilizando la media y desviación estándar, para cada una de las variables cuantitativas y se hallaron las frecuencias absolutas y relativas para las variables categóricas, procesando así los datos sociodemográficos, las respuestas de satisfacción y ponderación, así como las respuestas a las preguntas abiertas. Se utilizó el Alpha de Cronbach para el análisis de validez y fiabilidad, con un valor de 0.90 .

\begin{tabular}{|c|c|c|}
\hline \multirow{5}{*}{ Acceso vascular actual } & favi & $52,1 \%$ \\
\hline & injerto protésico & $3,2 \%$ \\
\hline & catéter permanente & $28,7 \%$ \\
\hline & catéter temporal & $8,5 \%$ \\
\hline & No contesta & $7,4 \%$ \\
\hline \multirow{5}{*}{ Acceso vascular en la 1 diálisis } & favi / injerto & $35,1 \%$ \\
\hline & injerto protésico & $4,3 \%$ \\
\hline & catéter permanente & $8,5 \%$ \\
\hline & catéter temporal & $42,3 \%$ \\
\hline & No contesta & $9,6 \%$ \\
\hline \multirow{3}{*}{ Problemas de acceso vascular } & si & $36,2 \%$ \\
\hline & no & $54,3 \%$ \\
\hline & No contesta & $9,6 \%$ \\
\hline \multirow{4}{*}{ Trasplantes anteriores } & 0 trasplantes & $85,1 \%$ \\
\hline & 1 trasplante & $11,7 \%$ \\
\hline & 2 trasplantes & $2,1 \%$ \\
\hline & 3 trasplantes & $1,1 \%$ \\
\hline \multirow{3}{*}{ Lista de trasplantes } & si & $37,2 \%$ \\
\hline & no & $60,6 \%$ \\
\hline & No contesta & $1,1 \%$ \\
\hline
\end{tabular}

Tabla 2. Características clínicas de los pacientes. 


\begin{tabular}{l|l|l|l}
\hline & \multicolumn{1}{c}{ CENTRO A } & \multicolumn{1}{c}{ CENTRO B } & \multicolumn{1}{c}{ CENTRO C } \\
\hline RATIO ENFERMERA/PACIE & $1 / 2-4$ & $1 / 5$ & $1 / 4$ \\
\hline RATIO MÉDICO/PACIENTE & $1 / 11$ pacientes & $1 / 25$ pacientes & $1 / 21$ pacientes \\
\hline RATIO AUXILIAR/PACIENTE & $1 / 13$ & $1 / 27-32$ & 2 meses \\
\hline FORMACIÓN ENFERMERÍA & 1 mes práctica & $2 \mathrm{~m}$ y curso seden & 2 turnos \\
\hline TURNOS & 4 turnos & 4 turnos & 1 con separaciones \\
\hline SALAS & 3 salas & 4 salas & Técnica aséptica \\
\hline CONEXION CATÉTER & Técnica estéril & Técnica aséptica & bocadillos \\
\hline CATERING & Bocadillos y yogures & bocadillos & camas \\
\hline UBICACIÓN DEL PACIENTE & camas & Sillones y camas & 1 grande/2 paciente \\
\hline TELEVISOR/PACIENTE & 1 pequeño /3 paciente & 1 grande/6 paciente & \\
\hline
\end{tabular}

Tabla 3. Datos descriptivos de los centros de Hemodiálisis.

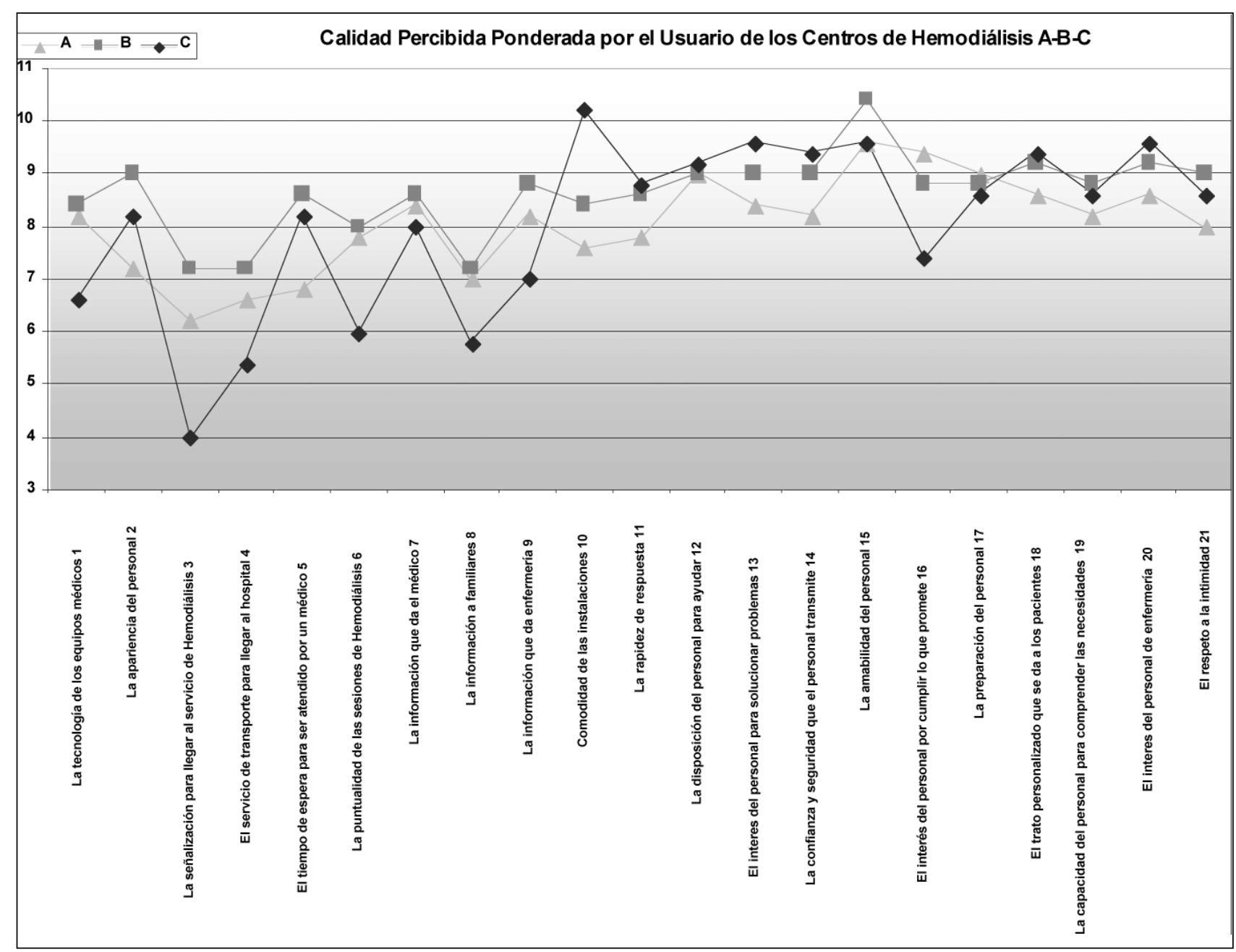

Tabla 4. Resultados de Satisfacción Ponderada de los centros A, B y C

Para determinar qué procedimiento de ponderación es más fiable, hallamos los factores de ponderación de cada centro por ambos procedimientos: La ponderación directamente puntuada según la importancia que el paciente atribuye a cada aspecto mediante la fórmula correspondiente ${ }^{7}$ y la ponderación mediante los coeficientes de correlación. Hemos expresado los resultados mediante un gráfico de línea con marcadores, para compararlos.

Para determinar cómo influyen las Características de los centros y las Características de los pacientes en los resultados de satisfacción, se realiza un análisis estadístico multivariante: la regresión lineal múltiple. Se 
utiliza el método forward (hacia adelante) que selecciona la variable independiente más explicativa de la variable dependiente. En cada ciclo incorpora una nueva variable independiente hasta que el modelo no mejora.

\section{Resultados:}

De los 148 pacientes participantes, el $71 \%$ fueron hombres. La media de edad fue de $67,1 \pm 14,1$ años, el tiempo medio en programa de Hemodiálisis fue de 3,5 años \pm 4 años (mucha variabilidad de datos). Un $85 \%$ de los pacientes estaban jubilados, un $12 \%$ trabajaba 0 se ocupaba del hogar y un 3,2\% no trabajaba. Un $62 \%$ tenía estudios primarios, un $15 \%$ no tenía estudios, un $14 \%$ acabó estudios secundarios y un $8.7 \%$ tenía estudios universitarios.

Satisfacción global con el servicio de HD: Un $62.8 \%$ se sienten muy satisfechos, el $36.2 \%$ se sienten satisfechos, el $1.1 \%$ se sienten poco satisfechos.

Recomendarían la unidad de HD: El $93.6 \%$ sin dudarlo frente al $5.3 \%$ tienen dudas y el 1,1 que no sabe o no contesta. Conoce el nombre del médico: El 93,6\% conoce su nombre mientras que el $6,4 \%$ no lo conoce.

Conoce el nombre de la enfermera: EL 95,7\% conoce el nombre de la enfermera, frente al $4,3 \%$ que no lo conoce.

En cuanto a los resultados del cuestionario SERVQHOS, la puntuación Global de Satisfacción manifestada por el usuario es del 4.3 sobre 5 (muy satisfecho). (Alpha de Cronbach de 0.90). Los Índices de Satisfacción Globales ponderados de cada centro son: Centro A:8, centro B: 8.6, centro C: 8 sobre 10.

En cuanto a la importancia que cada paciente da a los distintos aspectos del cuestionario, exponemos un listado con los resultados globales de los centros $A B C$ por orden de mayor a menor importancia:

\begin{tabular}{|r|l|c|}
\hline ITEMS & $\begin{array}{c}\text { En orden de importancia para } \\
\text { el paciente (abc) }\end{array}$ & $\begin{array}{c}\text { IMPOR- } \\
\text { TANCIA }\end{array}$ \\
\hline 15 & La amabilidad del personal & 9,6 \\
\hline 12 & La disposición del personal para ayudar & 9,5 \\
\hline 17 & La preparación del personal & 9,5 \\
\hline 13 & El interés del personal para solucionar problemas & 9,4 \\
\hline 14 & La confianza y seguridad que el personal transmite & 9,4 \\
\hline
\end{tabular}

\begin{tabular}{|c|l|c|}
\hline 18 & El trato personalizado que se da a los pacientes & 9,4 \\
\hline 16 & El interés del personal de enfermería & 9,4 \\
\hline 19 & El interés del personal por cumplir lo que promete & 9,3 \\
\hline 11 & La capacidad del personal para comprender las necesidades & 9,3 \\
\hline 21 & El respeto a la intimidad & 9,2 \\
\hline 2 & La apariencia del personal & 9,2 \\
\hline 9 & La información que da enfermería & 9,1 \\
\hline 7 & La información que da el médico & 9,1 \\
\hline 10 & Comodidad de las instalaciones & 9 \\
\hline 6 & La puntualidad de las sesiones de Hemodiálisis & 9 \\
\hline 1 & La tecnología de los equipos médicos & 8,8 \\
\hline 5 & El tiempo de espera para ser atendido por un médico & 8,7 \\
\hline 4 & El servicio de transporte para llegar al hospital & 8,4 \\
\hline 8 & La información a familiares & 8,2 \\
\hline 3 & La señalización para llegar al servicio de Hemodiálisis & 8 \\
\hline & & IPG: 9,1 \\
\hline
\end{tabular}

En el gráfico de barras (tabla 5) se observan las diferencias entre la calidad percibida por el usuario y la calidad esperada. Aquellos ítems que reflejen mayor diferencia entre calidad percibida y calidad esperada serán los que más deban mejorar (áreas de mejora).

Resultados de los dos métodos de ponderaración: Ponderación por Paciente y por Correlación (tabla 6).

En cuanto a la correlación de variables, utilizando la regresión lineal múltiple y relacionando características de centros y pacientes para verificar si éstas influían de algún modo en la satisfacción de los pacientes, comprobamos que:

- La satisfacción de 3 de los 21 ítems de la encuesta (La tecnología de los equipos médicos, la comodidad de las instalaciones y La disposición del personal para la ayuda) no depende de ninguna otra variable relacionada.

- La satisfacción de 5 de los 21 ítems de la encuesta (La apariencia del personal, La señalización para llegar a HD, La información que da Enfermería, La confianza y seguridad que el personal transmite, y El respeto a la intimidad) depende de las características del centro de $\mathrm{HD}$, como son: El número de salas de $\mathrm{HD}, \mathrm{El}$ ratio medico/paciente, El ratio enfermera/paciente, El ratio auxiliar/paciente, La formación de las enfermeras, La técnica de punción del acceso vascular, y de Los elementos de distracción. 


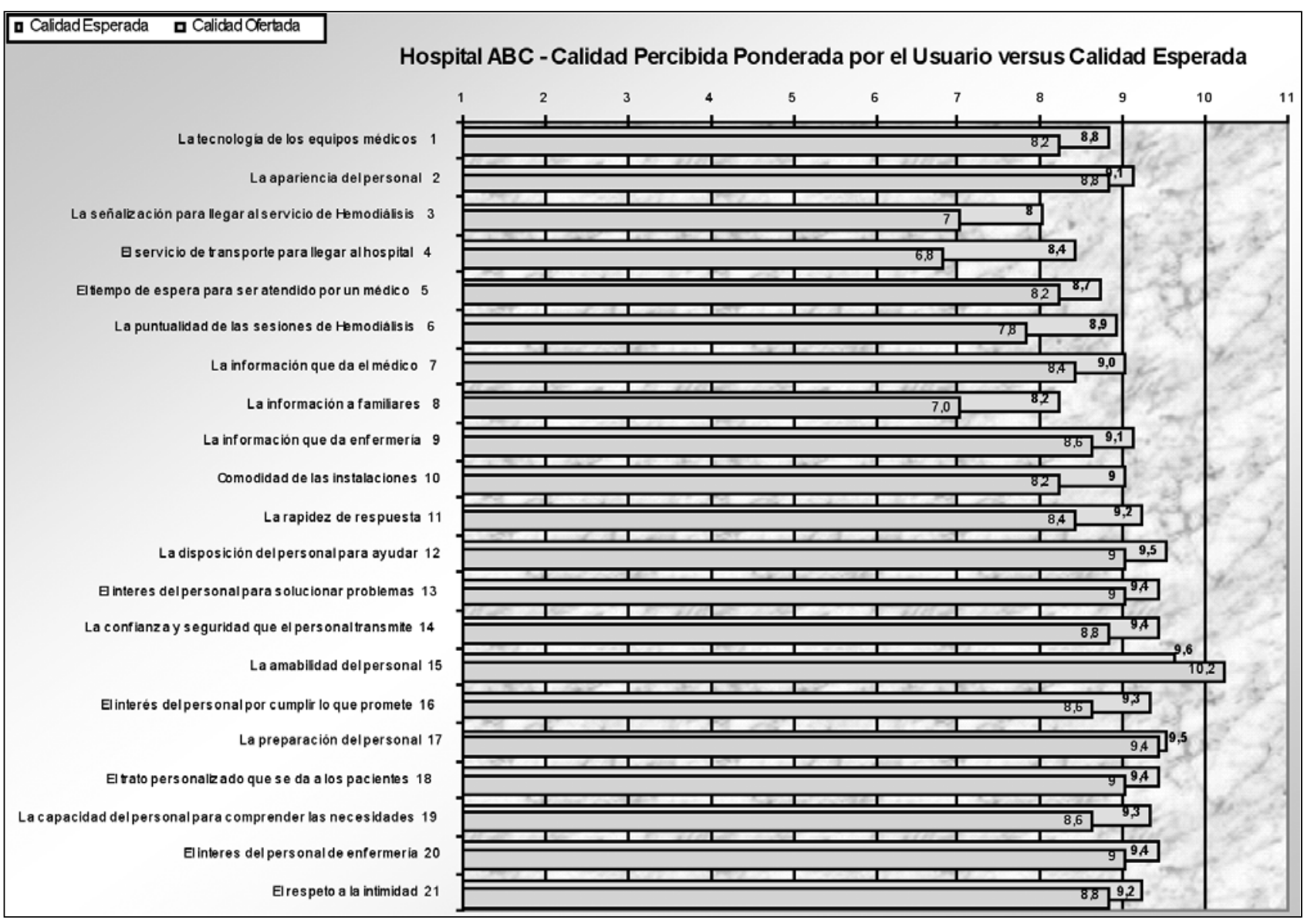

Tabla 5. Diferencia entre Calidad percibida y Calidad esperada.

口Ponderación por Correlación

La tecnología de los equipos médicos La apariencia del personal 2 La senalización para llegar al servicio El servicio de transporte para llegar al.

El tiempo de espera para ser

La puntualidad de las sesiones de.. La información que da el médico 7

Lainformación a familiares 8 La información que da enfermería Comodidad de las instalaciones 10 La rapidez de respuesta 11

La disposición del personal pare. El interes del personal pare. La confianza y seguridad que el. La amabilidad del personal 15 El interés del personal por cumplir lo..

La preparación del personal 17 El trato personalizado que se da a los La capacidad del personal para. Elinteres del personal de enfermería. El respeto a la intimidad 21

\section{ABÇ - Satisfaçción: Ponderación Pacjente \&. Pónderación Correlación}

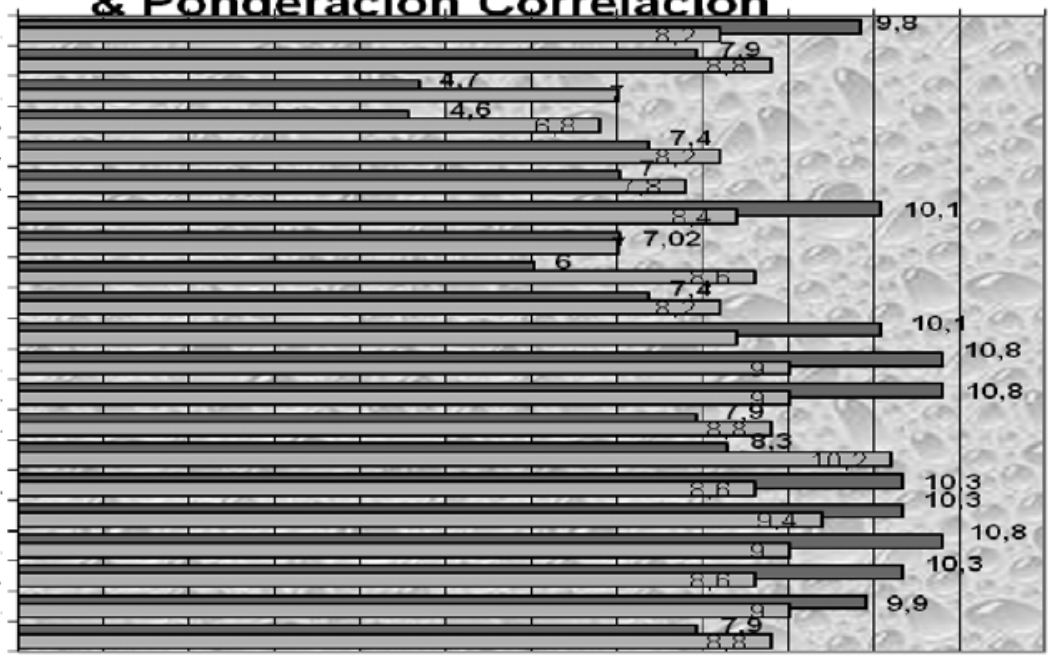

Tabla 6. Comparativa de los dos métodos de ponderación. 
- Hay otras 3 características de los centros (EI catering, La técnica de conexión-desconexión y EI número de turnos de HD) que influyen, respectivamente, en otros tres ítems de la encuesta de satisfacción: El tiempo de espera para ser atendido por un médico, La información que da Enfermería, y La información a familiares.

- Se comprueba, que un par de características que se consideran predictores de la calidad percibida: Conocer el nombre del médico y / o de la enfermera que les atiende, influyen en la satisfacción de dos de los ítems de la encuesta (La apariencia del personal y la preparación del personal).

- Se observa que el nivel de Satisfacción Global con el servicio de HD está fuertemente relacionado con la satisfacción de 16 de los ítems de la encuesta.

- Hay otras 11 características de los pacientes que influyen directamente en la satisfacción de uno o varios ítems de la encuesta.

\section{Discusión:}

Las características socio demográficas y clínicas de los pacientes de todos los centros son bastante similares, si bien es cierto que el centro $A$ tiene un menor porcentaje de FAVIs, esto podría ser debido a que es el centro de referencia en el que muchos pacientes realizan la diálisis urgente por primera vez. Sin embargo, podemos concluir que el perfil del usuario de los centros de HD de Tarragona es un hombre de edad avanzada 67 años y por tanto jubilado, con estudios primarios, que vive a menos de $20 \mathrm{~km}$ del centro de HD y que utiliza la ambulancia como medio de transporte. Se dializa por la mañana e hizo su primera HD mediante un catéter temporal y mediante una FAVI posteriormente. No tuvo problemas con su acceso vascular, pero si los tuviera, requeriría recambio de catéter o reparación quirúrgica. No espera ser trasplantado, se siente muy satisfecho con la atención recibida en su centro de HD y recomendaría la Unidad sin dudarlo. Conoce el nombre del médico y de la enfermera.

En cuanto al perfil del las Unidades de HD, debemos decir que las características de los 3 centros estudiados son muy diferentes, si bien es cierto que la mayoría de pacientes que se dializan lo hacen un centro que realiza 4 turnos de HD, con salas separadas en función de las serologías, con un ratio de 1 médico/25 pacientes, 1 enfermera/5 pacientes, 1 auxiliar/30 pacientes. Las enfermeras realizan 2 meses de prácticas tutorizadas en el centro y han realizado el curso SEDEN de Enfermera Nefrológica. La técnica de punción de favis es la habitual, con bisel hacia arriba. La técnica de conexión aséptica se realiza mediante una única enfermera que se prepara el campo estéril. El catering consiste en bocadillos. La ubicación del paciente es en sillones y como elemento de distracción principal tienen 1 televisor por cada 6 pacientes.

El nivel de calidad asistencial percibido por el usuario en la provincia de Tarragona es de 4,3 sobre 5 (muy satisfecho). El centro que obtiene mejores resultados ha sido el centro $B$, mientras que el centro $A$ i $C$ obtienen resultados muy similares.

Los aspectos mejor puntuados por los pacientes son, por orden: La amabilidad del personal, La preparación del personal, La disposición del personal para ayudar y solucionar problemas, EI trato personalizado y EI interés del personal de enfermería.

Los aspectos peor puntuados por los pacientes son, por orden: El servicio de transporte, La señalización para llegar al hospital y La información a familiares.

Por otro lado constatamos que los aspectos más importantes para el paciente son, por orden: La amabilidad del personal, La disposición del personal para ayudar, La preparación del personal, El interés para solucionar problemas, La confianza y seguridad que el personal sanitario transmite, El trato personalizado, y El interés del personal de enfermería. Mientras que los aspectos menos importantes para el paciente son, por orden: La señalización para llegar al servicio de HD, La información a familiares.

Concluimos que los ítems que más importan al paciente pertenecen al grupo de calidad subjetiva (ítems 11-21) y tienen menor importancia los aspectos relacionados con la calidad objetiva (ítems del 1-10).

Se han detectado áreas de mejora de la calidad asistencial de los centros $A, B$ y $C$, teniendo en cuenta la importancia que el paciente da a cada ítem y su puntuación de satisfacción obtenida mediante el 
cuestionario SERVQHOS. Los aspectos que más deben mejorar son El servicio de transporte para llegar al centro y La puntualidad de las sesiones de diálisis.

La ponderación mediante correlación lineal difiere bastante de la ponderación por el paciente. El método de la correlación lineal se basa en la asignación de factores de ponderación de forma aleatoria y no distingue entre baja importancia y poca satisfacción de un ítem, del mismo modo asocia importancia alta con mucha satisfacción de un aspecto. Esto plantea un problema cuando un aspecto es poco importante para el paciente pero lo puntúa alto porque considera que el servicio cumple muy bien con él, o cuando el aspecto es muy importante para el paciente y lo puntúa bajo porque considera que el servicio no cumple con él. Hemos despreciado este método y hemos optado por ponderar según la importancia que el propio paciente ha otorgado a cada ítem.

La satisfacción global del paciente depende de sus propias características (variables socio-demográficas y acceso vascular), del turno de hemodiálisis (M/T/N), del centro donde se dializa y del tipo de tratamiento que efectúa. Ya que, en el estudio multivariante, se comprueba que: tanto las características del paciente como las del centro de HD influyen en la satisfacción de uno o varios ítems de la encuesta. Al intentar discernir si las características de los centros influían en la satisfacción de los pacientes, hemos observado que los ratios personal/paciente no guardan relación con la satisfacción. Esto nos hace pensar que la satisfacción del usuario puede depender más de las cualidades del personal sanitario y de la interacción entre ambos, 0 de la relación de ayuda que establece entre enfermerapaciente. Podría ser interesante realizar un estudio de tipo cualitativo.

\section{Agradecimientos:}

Agradecemos a Rosa Aparicio, enfermera y profesora de la Universidad Rovira i Virgili, su ayuda en la estructuración de este trabajo, también a Carme Montcusí en lo referente a búsqueda bibliográfica. Nuestra gratitud a Eva Méndez Martínez, socióloga y técnico de investigación sociológica por sus sugerencias. Agradecemos la ayuda de Pilar Hernández Gutiérrez estadista y epidemióloga del Institut d'Investigació Sanitaria Pere Virgili. Agradecemos la colaboración proporcionada por las supervisoras y enfermeras de los Centros de Hemodiálisis y sobre todo gracias a los pacientes, sin su voz, este trabajo no hubiera sido posible.

\section{Bibliografía}

1. Calero García M J, Fernández Roa A J. Calidad Asistencial en Enfermería Hospitalaria. Granada: Fundación Index; 1995.

2. Quílez Castillo F. Calidad percibida por el enfermo. Un proyecto de futuro. Biseden 1994; (IV); 17-21.

3. Mira J J, Aranz J, Lorenzo S, Rodríguez-Marín J y Moyano S. Evolución de la Calidad Percibida por los pacientes en dos hospitales públicos. Psicothema 2001; 13 (4); 581-585.

4. Mira J J, Buil J A, Aranaz J, Vitaller J, Lorenzo S, Ignacio $E$, [et all]. ¿Qué opinan los pacientes de los hospitales públicos? Análisis de los niveles de calidad percibida en cinco hospitales. Gac Sanit 2000; 14 (4): 291-293. Disponible en: http://www.doyma.es.

5. Mira J J, Aranaz J, Rodríguez-Marín J, Buil J A, Castell M y Vitaller J. SERVQHOS; un cuestionario para evaluar la calidad percibida de la atención hospitalaria. Medicina Preventiva 1998; IV (4); $4^{\mathrm{a}}$ trimestre.

6. Hernández Meca ME, Ochando García A, Lorenzo Martínez S, Orbes Cervantes P, y López Revuelta $K$. Factores determinantes de la satisfacción del paciente en tratamiento renal sustitutivo. Rev Soc Esp Enferm Nefrol 2007; 10 (1); 6-13.

7. Palacios Gómez. Estrategias de Ponderación de la Respuesta en Encuestas de Satisfacción de Usuarios de Servicios. Metodología de Encuestas. Madrid: Universidad Complutense de Madrid 2002; 4 (2): 175-193.

8. Ministerio de Fomento. Como realizar el estudio de satisfacción. Madrid. Ediciones Mayo; 2005. 